\title{
Advancing knowledge on regulating tobacco products, Oslo, Norway
}

\section{Introductory presentations}

The meeting entitled "Advancing knowledge on regulating tobacco products", held in Oslo, Norway on 9-11 February 2000, was organised in response to a call by Dr Gro Harlem Brundtland, director general of the World Health Organization, for scientific evidence that could be used as the basis for regulating tobacco products. During the opening addresses, the then Norwegian Minister of Health, Mr Dagfinn Høybråten, and the WHO regional director for Europe, Dr Marc Danzon, both emphasised the need for sound evidence to be used as the basis for decisive action against tobacco. Mr Høybråten stressed the need for complementary national and global actions and urged that the output of the meeting be of use to the development of a product regulatory component of the Framework Convention on Tobacco Control (FCTC) and possible protocols.

The objectives of the meeting were based on an acknowledgement that comprehensive tobacco control policies and strategies were needed in all countries. The specific objectives of the meeting were to:

- exchange scientific information about tobacco product design and manufacture needed for regulation;

- define public health goals of regulation of tobacco products and how the concept of harm reduction fits within such goals;

- identify priority research areas required to advance regulating tobacco products;

- recommend whether a protocol on regulation should be developed as part of the FCTC.

BRIEF SYNOPSIS OF INTRODUCTORY PAPERS

A review of the epidemiology of tobacco and health, and current approaches to tobacco control, was presented by Derek Yach. Tobacco kills four million people annually worldwide and will kill 10 million a year by the 2020s. The epidemiological evidence highlights the need to assess the ultimate impact of control strategies on total mortality. A review of these strategies by Tapani Piha stressed the need for comprehensive national approaches that use effective measures able to reach all sectors of the population. Sustained investment in tobacco control over decades is required before results become evident.

Reporting on a recent meeting on tobacco product regulation held in Finland, Jack
Henningfield stressed that within a comprehensive approach, product regulation should play an increasing role. Product regulation should not undermine but rather should complement prevention and cessation strategies. He highlighted several areas where agreement is emerging:

- transnational approaches are vital;

- there is no "safe" cigarette;

- the addictiveness of nicotine and the toxicity of tobacco products provide the rationale for regulation;

- consumer information plays a fundamental role in product regulation strategies.

Judy Wilkenfeld highlighted the need for regulatory agencies to be able to evaluate and respond to changes in tobacco product design, and assess their health impact. The difficulties in doing this were outlined by Don Shopland, who also showed how public perception of risk was influenced by terms such as "low tar". There was little evidence of real health gains from such products. The intimate link between product design and smokers' behaviour was highlighted. This link explains why product design changes have failed to yield health gains (through compensatory mechanisms).

Clive Bates, Bill Rickert, and David Sweanor all highlighted major difficulties with existing testing methods when these are used to characterise smokers' exposure. Studies have shown that smokers adjust their smoking behaviour according to the availability of nicotine in smoke in order to achieve their desired dose. This effect, known as "compensation", is not emulated by any standardised smoking regime, such as the Federal Trade Commission (FTC) and International Organization for Standardization (ISO) methods. Testing methods able to evaluate the biological impact of existing and novel products are urgently needed.

Channing Robertson urged that testing methods be kept simple and not try to cover thousands of constituents of tobacco. $\mathrm{He}$ provided evidence demonstrating that cigarettes are designed to deliver optimal doses of nicotine masked by natural and added taste elements. Further, he believes that the onus of showing health gains from new products should be placed on the tobacco industry. The range of new and novel products, already at an advanced stage of development by the tobacco industry, was described. Some products attempt to provide 
very low levels of tar, nicotine, and side stream smoke.

Mitch Zeller reviewed the need for regulatory agencies to evaluate proactively the challenges posed by the ongoing debate surrounding exposure and harm reduction. $\mathrm{He}$ raised the potential unintended consequences of certain policies and urged that they be carefully considered before adoption.

David Sweanor recommended that a future regulatory framework should encourage wider use by smokers of "cleaner" forms of nicotine and disfavour the use of "dirty" forms.

\section{Working group discussions}

ANTICIPATING AND RESPONDING TO TOBACCO INDUSTRY TACTICS

Several well documented approaches used by the tobacco industry to oppose tobacco control in general were outlined. These are likely to be used in opposing tobacco product regulation. All approaches involve the tobacco industry spending large amounts of money and exerting its influence through multiple channels. Specific methods used include: lobbying; use of third parties; questioning the science and raising public doubt about health and addiction effects; warning governments about the cost of regulation; use of scientists with unproven ideas; dissemination and repackaging of legislative/regulatory problems from other countries; divide and conquer across bureaucracies, countries and public health groups; legal and media attacks on regulatory agencies; and constitutional challenges.

In developing countries, weaker knowledge about health impacts, weak regulatory and scientific capacity, and greater vulnerability of the media and politicians to the power of the industry meant that industry strategies were often more intense and successful.

When the tobacco industry cannot prevent new laws and regulations, it finds ways-for example, through voluntary agreements with governments and scientists-to ensure that regulations favour their interests.

PROACTIVE GLOBAL RESPONSES

- A global team of experts is needed to help countries deal with industry arguments.

- Global capacity within the public sector is currently inadequate to understand technical aspects of tobacco product regulation. Urgent attention should be given to developing expert capacity within the tobacco control community on technical issues relating to product design, manufacture, and technology.

- An international alert and response system linking media and experts should rapidly mount public responses to industry claims and critique. More effective use of the media should aim to reframe the debate away from tobacco industry claims.

- Clearinghouses for global knowledge on tobacco products are needed. They should maximise the use of information technology and draw on existing centres of excellence.

- Engagement with the tobacco industry should be discussed with the proposed expert group (see recommendations) and aim to achieve public health objectives. In the process of engagement the burden of proof for demonstration of less harm should be placed on the industry.

- National expert reports should be used globally. For example, the recent Royal College of Physicians (February 2000) and the pending FTC and Institute of Medicine reports will have wide implications.

- Adequate funding is needed for all aspects of product regulation, including research and development, and institutional development especially for developing countries

- A wider range of strategic partners should be involved in product regulation development and implementation. This could include any group that is willing to advance public health goals. As examples, such partners may include printers, pharmaceutical companies, large companies with workforces trying to quit, "ethical" corporations, and consumer groups.

\section{Objectives and principles of tobacco product regulation \\ OBJECTIVES}

- Prevent initiation of tobacco use and aim for a substantial and sustained reduction in tobacco related morbidity and mortality among smokers.

GENERAL PRINCIPLES

- Tobacco products (including smokeless tobacco) and nicotine delivery devices should be regulated on the basis of principles of premarket evaluation and placing the burden of proof for health claims on the tobacco industry.

- Research required to make health and/or safety claims should be paid for by the tobacco industry; full public disclosure of the methods, purpose and outcome of tobacco industry; research is essential.

- The intent of product regulation is to ensure that exposure reduction results in meaningful harm reduction.

- It was noted that FTC/ISO methods currently in use were not intended to measure the biological or epidemiological impact of tobacco products. New methods and protocols must be developed to measure the impact of tobacco products on an individual and population basis. ISO should be urged to ensure that its members recognise and adhere to the principle that ISO/FTC measurements and methods are used to monitor performance and not health impacts of tobacco products.

- Carefully planned communications programmes should be implemented in order to disseminate the implications of the outcome of tests. Harm/exposure reduction should be accompanied by intensive public education and increased public access to effective means of quitting.

- Developing countries should be encouraged and supported to "leapfrog" over the standard regulatory route followed in developed countries (go for the best from the start). 
Regional approaches are needed to complement national and global approaches; this will avoid the need for progress to depend upon moving on a country by country basis.

- The FCTC process and content should be exploited as a unique opportunity for giving focus and attention to product regulation.

\section{Recommendations}

These recommendations were developed by representatives from 20 countries from all WHO regions. While some recommendations suggest the need for additional research, countries are urged to act rapidly on the basis of a large body of existing knowledge. Exposure reduction policies must aim to achieve meaningful reductions in harm while avoiding decreased quitting or increased initiation.

(1) All countries need to introduce comprehensive tobacco control policies and strategies along the lines recommended by $\mathrm{WHO}$

These should be adequately financed and managed by institutions with a clear mandate for tobacco control. The emphases of policies should be to prevent initiation, increase the quit rate, and eliminate exposure to passive smoking. Within the context of a comprehensive policy, product regulation should be given explicit and urgent attention in order to reduce the health impact of tobacco use among smokers. Product regulation needs to apply to all forms of tobacco and nicotine products.

(2) Governments are urged, individually or at a regional level, to take the following actions:

- Evaluate and implement the most effective ways to achieve a unified regulatory framework for nicotine delivery products, including tobacco products, products for treating tobacco dependence, and novel nicotine delivery devices, whether or not these are based on tobacco products. Key terms of reference are to:

- maintain a primary focus on harm reduction;

- develop better measurement of the constituents and impact of tobacco products with the aim of substantially reducing their toxicity;

- promote international comparability;

- implement a premarket approval and post marketing surveillance system.

- Ban the use of misleading terms such as "light", "mild", and other words or imagery (including certain brand names) which have the aim or effect of implying a reduced health risk attributable to low tar or nicotine measurements on tobacco products and in advertising/promotional material.

- Remove tar and nicotine measures derived from ISO/FTC methods from packages. Warning labels should emphasise the addictiveness of tobacco products.

- Require tobacco manufacturers to disclose the contents, purpose and effects of constituents in all their products at regular intervals.
- Discontinue harm reduction strategies based on naïve interpretation of tar and nicotine yield measurements. This means abandoning the strategy of seeking lower nominal tar yields and, instead, finding approaches that genuinely reduce harm to nicotine users.

- Give urgent priority to studying the implications for harm reduction of reducing levels of nicotine and other possible addictive constituents in tobacco products over time.

- Give greater attention to increasing public access to the range of effective methods of treating tobacco dependence, including nicotine replacement therapies, and to encourage development and marketing of additional effective products.

- Develop and implement a comprehensive long term communication programme to accompany all the above actions that stresses that there is no safe cigarette and that nicotine addiction is a major public health concern.

(3) Research is needed to advance further progress

- Global tobacco control research needs to be better supported. Within such a plan, emphasis should be given to research to support product regulation within developing countries. Existing research institutions should work together to implement such an approach.

- In order to reduce the addictiveness of tobacco products, research is urgently needed to evaluate the benefits and/or hazards of reducing nicotine and other possible addictive constituents in tobacco products over time. Particular attention should be given in research to determining whether a threshold exists for addictiveness.

- Develop better measures, including biomarkers, to assess the health impact of the use of "less harmful" tobacco products in order to drive future regulatory actions. For exposure, a composite measure of toxicity is needed. In addition the unintended consequences of such products should be investigated.

- Expand behavioural research on how "cigarettes affect smokers" and how the population (of smokers and non-smokers) responds to claims about new products and to new packaging rules.

- Determine whether regulators should encourage the development of substantially less harmful nicotine delivery devices.

- Determine whether countries should forbid addition of all new additives and explicitly address the possibility of reducing the use of additives that make tobacco products more attractive and/or taste better.

- Evaluate how regulatory approaches developed for cigarettes could be adapted to cover all forms of tobacco use.

(4) International collaboration

- Establish, under WHO authority, an international expert group on tobacco and nicotine delivery devices. It needs to be well 
financed and have access to the best technical expertise available. It would guide international policy development with respect to product regulation and could facilitate access to scientific information needed for tobacco regulation. Its first task would be to study the recommendations of the Oslo meeting and recent scientific reports on the topic and make recommendations for action to WHO.

- A global team of experts, facilitated by WHO, is needed to help countries deal with industry arguments and development of tobacco product regulations.
- Ensure that issues related to product regulation are incorporated into the FCTC and related protocols.

- Communicate the outcome of this meeting to all appropriate national, regional and international agencies in an attempt to foster uniform approaches.

Following the meeting, Dr Goo H Brundtland announced that she will soon establish a WHO Scientific Advisory Committee on Tobacco Product Regulation.

\section{CALENDAR OF EVENTS}

Full details of events to be included in the calendar should be sent to fohn Weller, technical editor, BMA House, Tavistock Square, London WC1H 9fR, UK; john_weller@msn.com

World Congress on Lung Health 30 August-3 September 2000, Florence, Italy

Further details: European Respiratory Society. (Tel +41216 130202; fax +41216 172865; info@ersnet.org)

UICC Conference on Cancer Control Issues in the Year 2000

8-9 September 2000, Seattle, Washington, USA

Further details: GP Murphy, Cancer Research Center, Northwest Hospital, Pacific Northwest Cancer Foundation, Seattle, Washington, USA. (Fax +1 206 363 3196)

Ninth World Conference on Lung Cancer

11-15 September 2000, Tokyo, Japan Further details: International Communications Specialists, Inc, Sabo Kaikanbekkan, 2-7-4 Hirakawa-cho, Chiyoda-ku, Tokyo 102-8646. (Tel + 33263 6474; fax +33263 7077) 5th Brazilian-Luzitan congress on Senology

18-22 October 2000, Brasilia, Brazil

Further details: Brazilian Senology Society, CLN 201 BI. A Salas 109/111, 70832-510 Brasilia-DF, Brazil. (Tel +55 61224 3895; fax +55 61321 4875; bearzi@ bearzieventos.com.br)

National Lung Cancer Awareness Day 12 November 2000

Further details: Carolyn Clary-Macy, University of California, San Francisco/ Mount Zion Cancer Center, USA. (http:// cc.ucsf.edu/news/lung_ca_awareness.html or ALCASE at www.teleport.com/ alcase)

First Kuwait International Conference on Lung Cancer-Prevention and Treatment

13-15 November 2000, Kuwait

Further details: Kuwait Society fo Smoking and Cancer Prevention, Qadisaya, Block 1, Street 14, Bldg 2, PO Box 26733, Safat, 13128 Kuwait, Kuwait. (Tel + 965253 0120; fax + 965251 0137; futureks@ ncc.moc.kw)
UICC International Smokeout

16 November 2000

Further details: International Union Against Cancer, Geneva, Switzerland. (Fax +41 22 809 1810; education@uicc.org)

Sixth International Congress of Behavioral Medicine

15-18 November 2000, Brisbane, Queensland, Australia

Further details: Intermedia, PO Box 1280,

Milton, Queensland, 4064, Australia. (Tel +6173369 0477; fax +6173369 1512;

ICBM@im.com.au; www.icbm2000.conf.au)

World Assembly on Tobacco Counters Health-WATCH 2000

4-8 December 2000, New Delhi, India Further details: Dr Vimal Arora, 509-B Arita Vihar, 110044 New Delhi, India. (Tel +91 11694 4551; fax +91 11694 4472; varora@ndf.vsnl.net.in)

92nd American Association for Cancer Research Annual Meeting 24-28 March 2001, New Orleans, USA

Further details: American Association for Cancer Research, Public Ledger Building, Suite 816, 150 South Independence Mall West, Philadelphia PA 19106-3483, USA. (Tel +1 215440 9300; fax +1 215351 9165; meetings@aacr.org) 